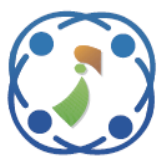

\title{
A Hybrid Linear-Quadratic Estimation (LQE) Technique for Channel Estimation in MIMO-OFDM System
}

\author{
Chetana Reddy ${ }^{1 *} \quad$ Virendra Shete ${ }^{2}$ \\ ${ }^{1}$ Department of Electrical and Computer Engineering, \\ Visvesvaraya Technological University, belagavi, Karnataka, India \\ ${ }^{2}$ Department of Electrical and Computer Engineering and Vice Principal MITCOE, \\ MITADT University, Pune, India \\ * Corresponding author's Email: chetana.ec@gmail.com
}

\begin{abstract}
The rapid development of wireless communication technology makes Orthogonal Frequency Division Multiplexing (OFDM) technology and Multiple Input Multiple Output (MIMO) technology, key candidates for the next generation mobile communication system. OFDM system has high frequency utilization and effectively resists multipath and narrowband interference. MIMO technology can effectively improve system capacity and system separation. This paper presents a hybrid Linear-Quadratic Estimation (LQE) technique for Channel Estimation in MIMO-OFDM system. In channel estimation, the noise covariance matrix (Q) plays a key role. In general, the value of $\mathrm{Q}$ depends on the previous state values. In a dynamic system, the previous values are not always known. The proposed hybrid model uses two techniques to address this issue. When the previous state values are known, Fast Adaptive Hybrid - Linear Quadratic Estimation (FAH-LQE) is used and when the previous state values are unknown Discrete Multivariate Hybrid - Linear Quadratic Estimation (DMH-LQE) is used. This enables the channel estimation to perform effectively in both scenarios. The FAH-LQE method uses covariance channel noise estimation equations and updates the threshold limit. DMH-LQE finds previous values using discrete generate matrix. The switching between FAH-LQE and DMH-LQE, based on the availability of previous state values, has increased the performance of the system effectively. The proposed algorithm produced lower BER values. For a mobility of $55 \mathrm{~km} / \mathrm{h}$ at SNR 15 , BER values of proposed algorithm are $22 \%, 10 \%, 7 \%, 6 \%$, and $3 \%$ lower than existing methods namely CE with and without EEP-Kalman tracking, perfect channel estimation, FAH-LQE, and DMH-LQE respectively. Similarly for a mobility of $115 \mathrm{~km} / \mathrm{h}$ at SNR 15, BER values of the proposed algorithm are $45 \%, 35 \%, 20 \%, 15 \%$ and $5 \%$ lower than above mentioned existing methods namely CE with and without EEP-Kalman tracking, perfect channel estimation, FAH-LQE, and DMH-LQE respectively. The SER has been reduced by $5 \%$ when no compensation method is applied. By using the proposed method, the Peak to Average Power Ratio (PAPR) has also reduced.
\end{abstract}

Keywords: OFDM, MIMO, Channel estimation, Hybrid linear-quadratic estimation (LQE), Fast adaptive hybrid linear quadratic estimation (FAH- LQE), Discrete multivariate hybrid - linear quadratic estimation (DMH- LQE).

\section{Introduction}

The great challenge of wireless communication systems is to provide a high transmission speed and to offer a good quality service. In recent years, there has been an increase in the demand for services like wireless broadband, video conferencing, cellular telephone etc., [1]. The development of such technologies face two significant problems: i. the frequency spectrum is a scarce and limited resource,

ii. transmission conditions are hostile due to fading caused by the environment and interference caused by the presence of other users.

Thus, technologies that present spectral efficiency and transmission reliability have become essential. OFDM modulation technique uses multiple orthogonal carriers which are superimposed on one 
another [2]. It became popular in the 90s with the advent of high-capacity processors. OFDM is becoming a popular technique for the transmission of broadband signals over wireless channels. OFDM transforms a frequency selective channel into a parallel set of sub-channels that facilitate signal transmission. Currently, OFDM is used in various broadband transmission standards [3], such as Digital Audio Standards (DAS), Digital Audio Broadcasting (DAB), digital television, Digital Video Broadcasting Terrestrial (DVB-T) and Integrated Service of Digital Broadcasting Terrestrial (ISDB-T), in addition to the WI-FI and WI-MAX standards in wireless networks [4]. When the number of carriers in the OFDM system increase, the orthogonality between the OFDM carriers is easily destroyed. This gives rise to Inter-Carrier Interference (ICI), which reduces the performance of the system.

\subsection{Interference in MIMO OFDM systems}

In the signal transmission process, the signal is affected by various parameters such as environment, fading caused by obstacles, varying time delays and synchronization errors. Time synchronization errors can cause Inter Symbol Interference (ISI), and frequency synchronization errors can cause InterCarrier Interference (ICI) [5]. In addition, because of the use of multi-antenna technology, inter-symbol interference and inter-carrier interference are becoming more severe and are affecting the system performance [6]. Therefore, the receiving end needs to obtain precise and accurate Channel State Information (CSI). With a good understanding of channel characteristics, one can effectively overcome interference and distortion. The accuracy of CSI directly affects the wireless communication and the overall performance of the communication system. Hence channel estimation is a reliable option to improve the performance of wireless communication.

When the symbol period in the time domain of OFDM system is extended, the interval between subcarriers is reduced. This phenomenon makes the communication system sensitive to phase noise. Phase noise can cause Common Phase Error (CPE) and inter-subcarrier interference in OFDM signals, thereby reducing the system transmission performance [7].

Some of the channel estimation techniques currently being used for MIMO-OFDM systems are Block Type Channel Estimation (BTCE) and Pilot Assisted Channel Estimation (PACE) [8]. BTCE uses OFDM special symbols called preamble which are sent periodically. All carriers are used to send information known to the receiver to estimate the channel [9]. Whereas, PACE sends information known to the receiver only on some carriers (usually evenly spaced in frequency).

This paper presents a novel Hybrid LinearQuadratic Estimation comprising of FAH-LQE and DMH-LQE channel estimation techniques. The MIMO-OFDM model analyses the availability of the previous stage parameters and selects the appropriate method to perform channel estimation. This results in a lower bit error rate. The remainder of this paper is organized as follows. Section 2 presents the literature survey. Section 3 describes the Linear-Quadratic Estimation Method. Section 4 presents the proposed hybrid channel estimation method. Section 5 presents the experimental results, followed by a conclusion and references.

\section{Literature review}

A filtering method for MIMO-OFDM systems is proposed in [10] to reduce the bit error rate. In these systems, the Kalman filtering technique implements to estimate the channel effectively, compared with its actual value. The simulation results show that the improved SNR using a two-phase Kalman filter in MIMO-OFDM systems.

In [11], authors proposed a new $\mathrm{CE}$ scheme to improve the efficiency of the MIMO-OFDM system suitable for ultra-speed data transfer in which the values of the bit rate of error are mitigated. In addition, this proposed system revisions the degree to which the original channel volatility and the MIMO (CT) value were improved using the discrete multivariate hybrid linear quadratic assesses method.

Several mixed Particle Filtering (PF) methods, including noise statistics and channel OFDM systems are presented in [12]. In the investigated problem with Carrier Frequency Offset (CFO), the measurement equation is somewhat non-linear. Consequently, it might not be possible to get a Machine Learning (ML) solution. Although numerical methodology based or asymptotically optimal performance-based methods have been offered for different problems, the numerical methods have some toxic characteristics. The authors proposed several mixture cost-reference particle filtering (CRPF) approaches for joint estimation of Channel Impulse Response (CIR) and CFO. A couple of them can be applied without the knowledge of both state and measurement noise statistics.

The authors discussed phase noise and joint channel estimation for MIMO-OFDM systems where independent oscillators equip each antenna [13]. Novel placement of nulls and pilots have been used in the preamble and data portion of the MIMO- 
OFDM frame to compute the common phase error and the channel impulse response.

In research article [14], a digital Self Interference (SI) cancellation method using an original estimator of linear SI channel is proposed. A series of quadratic programming issues resolve and derives an optimally weighted matrix from obtaining the minimum residual SI power. The proposed method shows more proficiency than the previous Least Square (LS) and Minimum Mean Square Error (MMSE) estimator.

A new pilot length minimization MIMO-OFDM Channel Estimation (CE) scheme is proposed in [15]. The scheme is designed based on new pilot bits from type I Hadamard matrices (HM-P) to handle orthogonal and diagonal constant matrix structures. In addition, they have assessed the magnitude of the channel response reaction as the sum of the multipath. Additionally, they have proposed estimating channel system adapted to derive the original channel flow and its doppler stage during the payload. A fast adaptive hybrid linear quadratic estimation (FAHLQE) filter for surveillance of the MIMO channel is used. Rayleigh distribution is considered while performing the experimental simulation in the MATLAB environment.

The author discusses various channel estimation techniques for MIMO-OFDM systems in [16]. The main challenges that occur while designing the soft computing-based channel estimation methods have been highlighted. The author has concluded that extensive work is required for the channel estimation methods to work efficiently in realistic environments.

In [17], authors have been investigated the MISO, MIMO, and SISO channel estimation techniques for OFDM systems. The comb-type and block-type pilot arrangements and different interpolation techniques have been compared to estimate the optimum performance. Compared with the other interpolation techniques, FFT showed the best performance, and MMSE outperforms the LS method.

The optimized filter framework and approximate MIMO OFDM technology via connected channel installation and management is proposed in [18]. The work presented is a simulation of 64 MIMO-OFDM sub-carriers with digital modulation technologies like BPSK and XD-4000. AWGN channel BER efficiency has been observed to be superior to Rayleigh and Rician channel from the findings.

In paper [19], authors have been implemented a novel reduced equalizer technique to solve a semiblind sparse channel/data calculation issue. A new configuration of a 'full' equalization array allows for data/channel estimation of fewer OFDM blocks generated. The reduced equalizer further reduces the number of OFDM blocks. The novel method may thus be regarded as the blind Bayesian method.

A framework was proposed in [20], which develops a joint Sparse double selective channel measurement with a symbol detector. The simulation is based on the Riccati equation of the $\mathrm{KF}$ performance benchmark. In addition, the Asymptotic Mean Square Error (MSE) is also acquired from Bayesian Cramer-Rao bounds. The simulation results demonstrated quality improvement in terms of either MSE or BER and validation of the analytics.

A phase rotation technique to limit the imaginary interference leakage by containing the interference within the signal imaginary part is proposed in [21]. The practical implementations, multiple sub-bands and generated solutions have discussed. According to literature, authors proposed different channel estimation methods which exhibits better error reduction. However, these research works are dependent on previous known values of the system. The previous know values play a major role to estimate the future prediction values in channel estimation algorithms. When previous known values are unknown, the existing algorithms fails to obtain better results. In this work, a hybrid estimation algorithm is proposed to overcome this issue. In proposed algorithm, the previous known values are generated by using discrete process co-variance matrix. The complete explanation of the proposed algorithm is described in Section 4.

\section{Linear-quadratic estimation method}

The Kalman filter keeps track of the estimated state of the system and the variance or uncertainty of the estimate. The estimate is updated using a state transition model and measurements. In statistics and control theory, Kalman filtering, also known as linear-quadratic estimation (LQE), is an algorithm that uses a series of measurements observed over time, containing statistical noise and other inaccuracies. Thus, it produces estimates of unknown variables that tend to be more accurate than those based on a single measurement alone, by estimating a joint probability distribution over the variables for each time frame.

The algorithm works in a two-step process. In the prediction step, the Kalman filter produces estimates of the current state variables, along with their uncertainties. Once the outcome of the next measurement (necessarily corrupted with some amount of error, including random noise) is observed, these estimates are updated using a weighted average, with more weight being given to estimates with higher certainty. The algorithm is recursive. It can run in real time, using only the present input 
measurements and the previously calculated state and its uncertainty matrix; no additional past information is required.

\subsection{Kalman method}

In this research, AWGN channel is considered for experimentation. Channel estimation is performed by using Kalman method. This method simply segregates into two steps: first step is to measure the predicting equations and second step is to calculate the measurement equations. In step two, the kalman gain has been measured at each iteration until the noise is supressed. The channel estimation by using kalman method as follows:

Step 1: Predicting Eqs. (1) and (2)

$$
\begin{gathered}
G_{k+1}^{T}=A G_{k}^{T} \\
P_{k+1}^{T}=A P_{k} A^{T}+Q
\end{gathered}
$$

Step 2: Measurement Eqs. (3) and (4)

$$
\begin{gathered}
K_{k}=P_{k}^{-}\left(D_{k}^{T}\right)^{H}\left(D_{k}^{T} P_{K}^{-}\left(D_{K}^{T}\right)^{H}+\sigma^{2}\right)^{-1} \\
G_{k}^{T}=G_{k}^{-T}+K_{k}\left(X_{k}^{T}-D_{k}^{T} G_{K}^{-T}\right) \text { and } \\
P_{k}=\left(I_{N_{t} N}-K_{k} D_{k}^{T}\right) P_{K}^{-}
\end{gathered}
$$

Where,

the prior and posterior estimate error covariance matrices represent as $P_{K}^{-}$and $P_{k}$ respectively,

the Kalman gain is denoted as $K_{k}$,

$\sigma^{2}$ refers to the noise covariance for the process and measurement models,

$I_{N_{t} N}$ indicates the identity matrix with the size of Nt $X N$, A denotes the channel transition matrix.

All these parameters have tuned properly for an excellent CT mechanism. behind the main title.

\section{Proposed hybrid channel estimation method}

The proposed channel estimation technique is a combination of FAH-LQE and DMH- LQE. This section presents the detailed implementation of the proposed method.

Consider the initial state of the channel estimation system as in Eq. (5).

$$
X_{0}=\text { State matrix }
$$

$P_{0}=$ Process co-variance matrix (it represents error in the process)
The present state of the system is marked as $k$ and the previous state is represented as $(k-1)$. The state variables are thus denoted as Eq. (6).

$$
\begin{gathered}
X_{k-1}=\text { previous predicted state variable } \\
P_{k-1}=\text { previous predicted process co-variance } \\
\text { variable }
\end{gathered}
$$

Then based on initial state $\&$ previous state, the new state is predicted as Eqs. (7) and (8).

$$
\begin{gathered}
X_{k p}=A X_{k-1}+B \mu_{k}+w_{k} \\
P_{k p}=A P_{k-1}+A^{T}+Q_{k}
\end{gathered}
$$

Where,

$X_{k p}$ is the predicted state matrix,

$P_{k p}$ is the predicted process co-variance matrix,

$\mu=$ control variable matrix,

$w=$ predicted state noise matrix,

$Q=$ process noise covariance matrix,

$A \& B \Rightarrow$ Adaption matrix.

The variations in channel are depended on the prediction matrix $X_{k p}$. Here the control variable matrix $\mu$ plays a major role in the variation of the channel. In this model the mobility of the user equipment is considered as control variable.

The input measurement is carried out using the Eq. (9)

$$
Y_{k}=c X_{k}+Z_{k}
$$

$Z_{k}=>$ measurement noise,

$C=>$ adaption matrix,

$Y=>$ measurement of state.

Once the new state is applied, these two, predicted state \& measurement input, are assigned to the new measurement \& Kalman gain.

The new measurement \& Kalman gain is calculated from Eqs. (10) to (12) respectively.

$$
\begin{gathered}
K=\frac{P_{k p} H}{H P_{k p} H^{P}+R} \\
X_{K}=X_{K p}+K\left[Y-H X_{K p}\right]
\end{gathered}
$$

Then

$$
P_{K}=(I-K H) P_{K p}
$$

Where,

$k=$ Kalman gain,

$R=$ sensor noise covariance matrix,

$I=$ Identity matrix. 


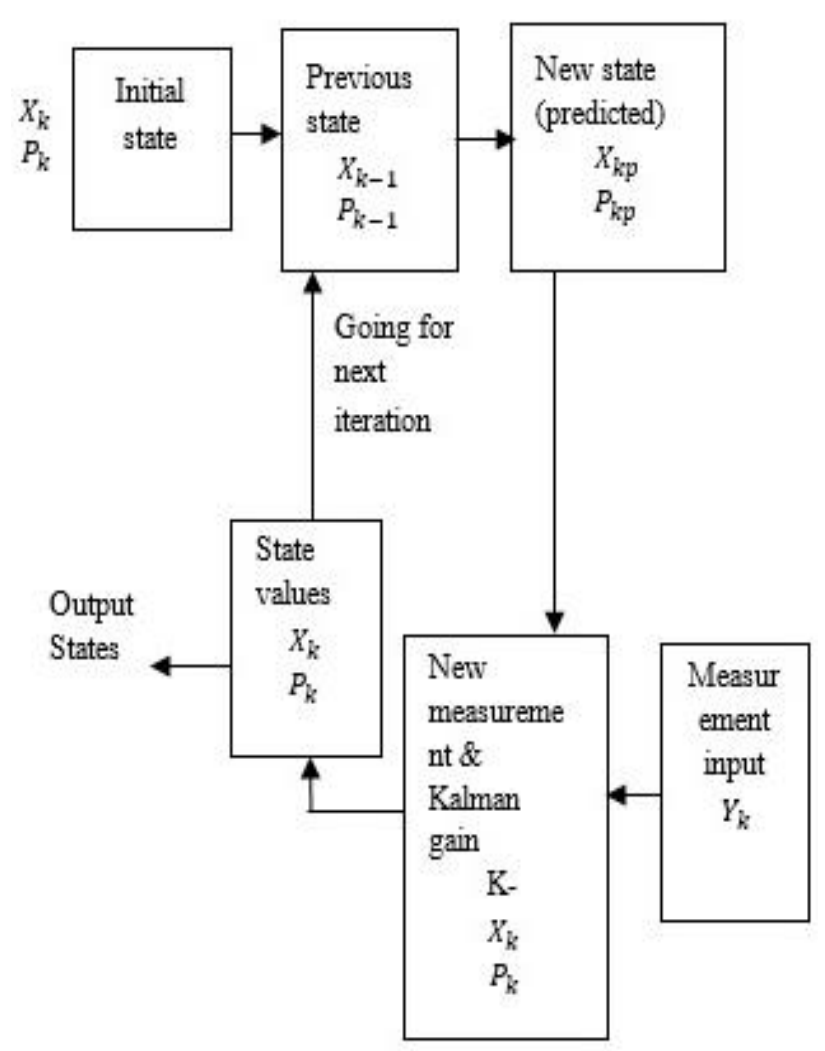

Figure. 1 Working principle of Hybrid-LQE method

Fig. 1 represents the functional blocks of the Hybrid-LQE method. The initial state values $X_{k}$ and $P_{k}$ are considered as input values. In the second block, the previous state values are measured using input state vales from the initial block. Based on the initial state and previous state values, the new state values have been calculated. On the other hand, the measurement values, also known as original values, are taken from the system. The next block deals with new measurement and Kalman gain block, which plays an important role. The new measurement and Kalman gain values are measured by using measurement input and new state values. Finally, the new state values will be obtained as the output of the Kalman gain block. However, these state values are not considered as output state values due to threshold condition. Once the threshold condition is verified, then, based on the threshold condition, the new state values are treated as output or treated as the input for the next iteration.

The block diagram of the Hybrid-LQE OFDM system model is depicted in figure 2 . The channel estimation and tracking are performed at the receiver end. The modulated received data with cyclic prefix is passed through the TD-channel estimation block. The cyclic prefix will be removed in the channel estimation block, and then corresponding data is passed through the Hybrid channel estimation block.

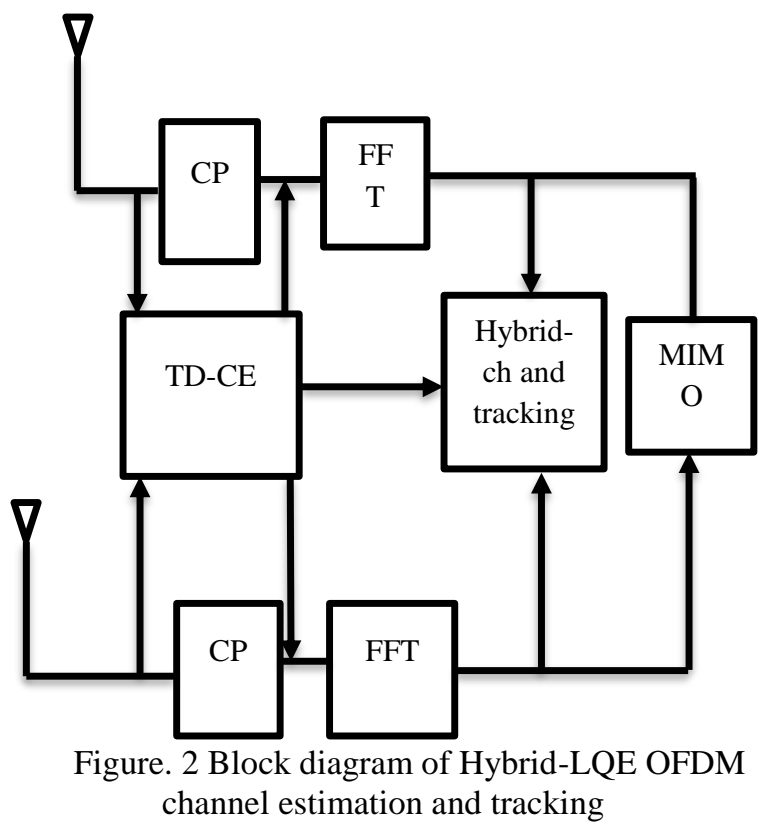

The hybrid channel estimation block receives two sets of data inputs: the first one without applying FFT data and the second is data with applying FFT operation. The working operation of hybrid channel estimation, which is discussed in Fig. 1 will be performed. Based on the output of the hybrid channel estimation block, error rate will be reduced.

The noise co-variance matrix plays important role to minimize the error in process co-variance

Matrix which will provide the better prediction state values in estimation.

- Let's take process co-variance matrix Eq. (13).

$$
P_{k p}=A_{k-1} A^{T}+Q_{k}
$$

Here $Q$ is depends on the previous state values $\left(P_{k-1}\right)$.

Algorithm is split in to two parts (cases).

1. Case: if previous values are known.

2. Case: if previous values are unknown.

Our Algorithm will work for both cases.

\section{Case 1:}

1. If the previous values known then FAH algorithm will execute as follows.

FAH - is split in to two states

2. Update the covariance of channel noise $Q_{v}(n)$ with known previous state values Update Eq. (14).

$$
Q_{v}=(1-d) Q_{v}(n)+d X Q_{u}(n)
$$

Where $\mathrm{d}=$ is loss factor. 
If limits the filtering memory length as Eq. (15).

$$
D=\frac{1-b}{1-b^{t+1}}
$$

Where,

$\mathrm{b}=0.9$ to 0.99 range,

$Q_{v}(n)=>$ Previous state,

$Q_{u}(n)=>$ Current state.

3. Update the threshold values (T)

Why because we need to find out the $Q_{u}(n)$ values are a noise or not.

The threshold will beside the $Q_{u}(n)$ acceptable noise or /not.

\section{Condition}

If $Q_{u}(n)$ is less than threshold then if is not consider then update the co-variance matrix in Eq. (16).

$$
T=(1-d) \times T+d \times Q_{u}(n)
$$

\section{Case 2:}

If previous values unknown and incoming state values consists dynamic in nature. Then DMH will execute in DMH if " $Q$ " is unknown

They it will generates from the describe process covariance matrix in Eqs. (17) and (18).

$$
X_{k+1}=F * X_{k}
$$

Where $*$ denoted as multiplication between two variables.

$$
P_{k+1}=F P_{k}+Q
$$
gain.

Then compute measurement data $\&$ then Kalman

\subsection{Phase noise estimation}

As mentioned in the introduction section, phase noise is generated in the OFDM system, CPE distortion which will seriously degrade system performance and it must be compensated. In an OFDM system, the output of transmitted signal $s(n)$ can be expressed as Eq. (19).

$$
s(n)=\frac{1}{N} \sum_{k=0}^{N-1} X(K) e^{\frac{2 \pi}{j N} k n}
$$

Where $n=0,1,2, \ldots \ldots . . N-1$.

$X(K)$ represents the signal modulated on each subcarrier of the OFDM symbol, where $K=$ $0,1,2, \ldots \ldots . . N-1, N$ is the subcarrier of the OFDM system. $s(n)$ is the output of the transmitter. Similarly, the input signal of OFDM receiver signal $y(n)$ can be expressed as in Eq. (20).

$$
y(n)=s(n) \odot h(n) e^{j \varnothing(n)}+m(n)
$$

Where,

$\odot$ represents the convolution symbol,

$h(n)$ is the sampling of the channel impulse response,

$\emptyset(n)$ is phase noise,

$m(n)$ is additive noise, and its variance is $\delta 2$.

The phase compensation of the model can be derived from the following equations. The time domain of $y(n)$ at $q^{\text {th }}$ receiver antenna the OFDM receiver signal $y_{q}(n)$ in the presence of phase noise can be expressed as Eq. (21).

$$
\begin{array}{r}
y_{q}(n)=e^{j \theta_{q}^{[r]}(n)} \sum_{m=1}^{N_{t}} h_{q, m}(n) * e^{j \theta_{m}^{[t]}(n)} s_{m}(n)+ \\
M_{q}(n)(21)
\end{array}
$$

Where $\theta_{q}^{[r]}(n)$ and $\theta_{q}^{[t]}(n)$ are phase noises of the $q^{\text {th }}$ receiver and $m^{\text {th }}$ transmitter. $N_{t}$ is the number of transmitter antennas.

In this model, the DFT based compensation is used. After applying the DFT, the received signal becomes at $q^{\text {th }}$ antenna and at $k^{\text {th }}$ subcarrier can be expressed as Eq. (22).

$$
Y_{q}(k)=\sum_{m=1}^{N_{t}} H_{q, m}(k) \underbrace{P_{m}^{[t]}(k) S_{m}(k)}_{C P E_{q, m}}
$$

Where $P_{q}^{[r]}(k)$ and $P_{m}^{[t]}(k)$ are the fourier cofients givien by $P_{q}^{[r]}(k)=\frac{1}{N_{c}} D F T\left\{e^{j \theta_{q}^{[r]}(n)}\right\}$ $P_{m}^{[t]}(k)=\frac{1}{N_{c}} D F T\left\{e^{j \theta_{m}^{[t]}(n)}\right\}$ of the $q^{t h}$ receiver and $m^{\text {th }}$ transmitter respectively. $N_{c}$ is the number of subcarriers. $C P E_{q, m}$ is the common phase error of the $q^{\text {th }}$ receiver and $m^{\text {th }}$ transmitter.

The flow chart of the Hybrid-LQE method is an iterative model and it is depicted in Fig. 3. The flow of the algorithm is similar to the working blocks which is explained in previous section and shown in Fig. 1.

The main difference of the working blocks and flow chart is explained here. The flow of algorithm works based on the previous state values, which can predict the noise covariance matrix. If the previous state values are known then the FAH algorithm will be executed or else the DMH algorithm will be performed. The switching activity is based on the previous state values. The remaining process description is described to the working blocks at Fig. 1 . 


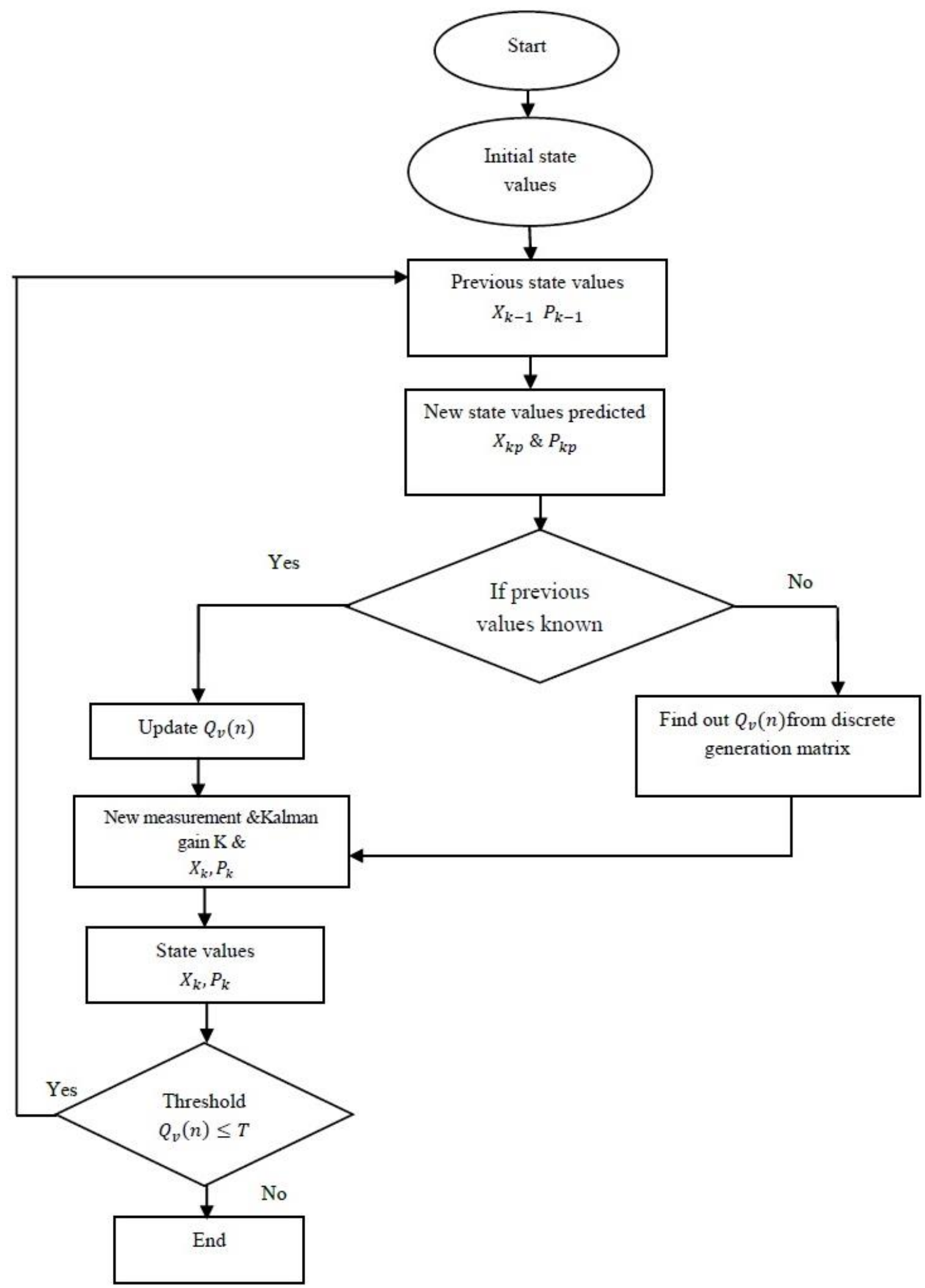

Figure. 3 Flow chart of Proposed Hybrid-LQE method

\section{Results}

This section describes the simulated results performed in MATLAB environment for obtaining the proposed MIMO-CE-CT. Table 1 disclosed that the parameters utilized in the simulation. The mean squared error (MSE) of the channel estimates compared to the original channels is also studied. 
Table 1. Simulation parameters

\begin{tabular}{|c|c|c|}
\hline Symbol & Parameter & Value \\
\hline$f_{c}$ & Carrier frequency & $2.1 \mathrm{GHz}$ \\
\hline $\mathrm{R}$ & Data rate per Tx & $600 \mathrm{kbps}$ \\
\hline$\mu$ & $\begin{array}{c}\text { Vehicle mobile } \\
\text { velocity }\end{array}$ & $\begin{array}{c}55 \mathrm{~km} / \mathrm{hr} \text { and } \\
115 \mathrm{~km} / \mathrm{hr}\end{array}$ \\
\hline $\mathrm{L}$ & Length of CIR & $\begin{array}{c}3(\text { equally spaced } \\
\text { by one sample })\end{array}$ \\
\hline $\mathrm{N}$ & FFT/IFFT size & 512 \\
\hline$f_{l}$ & Frame length & $\begin{array}{c}200 \text { OFDM } \\
\text { symbols }\end{array}$ \\
\hline$N_{r} \mathrm{X} N_{t}$ & $\begin{array}{c}\text { MIMO } \\
\text { configuration }\end{array}$ & $\begin{array}{c}(3 \times 2): 3 \mathrm{Tx} \& \\
2 \mathrm{Rx} \text { antennas }\end{array}$ \\
\hline
\end{tabular}

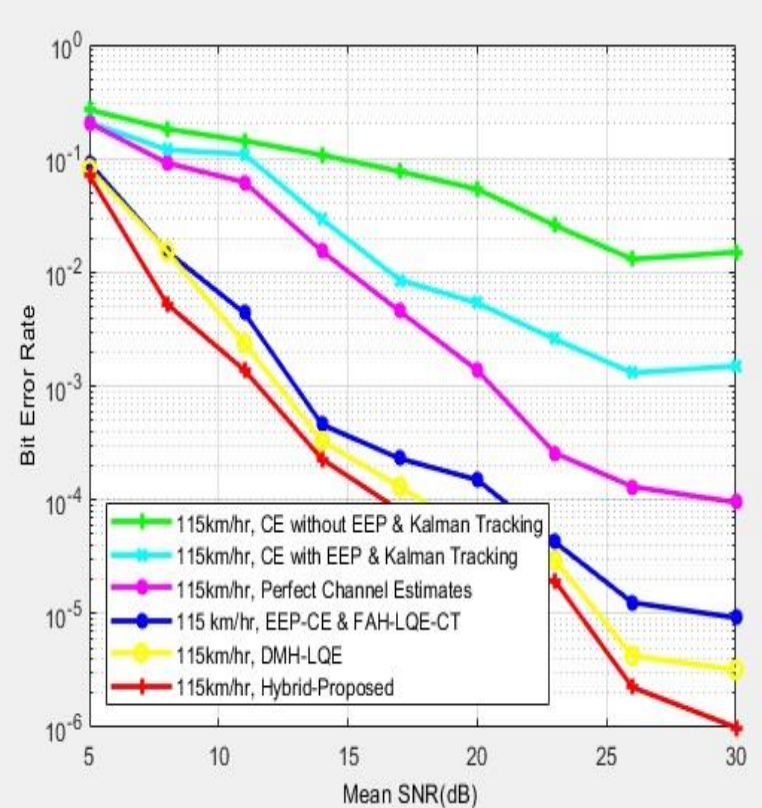

Figure. 4 BER performance of $(3 \times 2)$ MIMO-OFDM system with $\mu=115 \mathrm{~km} / \mathrm{hr}$ channels and $\mathrm{R}=600 \mathrm{kbps}$

MSEs are accepted at the total pilot bits mid-length those are sent per each Tx antenna.

The bit error rate (BER) system performance of a (3x2) MIMO-OFDM system is shown in Fig. 4 and 5 for $115 \mathrm{~km} / \mathrm{hr}$ and $55 \mathrm{~km} / \mathrm{hr}$ respectively.

The prosed Hybrid-LQE method is compared with other channel estimation methods with and without Efficient Enlargement of Pilot (EEP) which reported in [15, 22]. This EEP is a method to find the channel variation information and this can be used to measure the length of channel impulse response (CIR). In Fig. 4, the velocity of the user equipment (UE) such as mobile devices can be considered 155 $\mathrm{km} / \mathrm{hr}$. In this scenario the results are compared at SNR 30 and the BER values of the proposed algorithm (Hybrid LQE): 45\%, 35\%, 20\%, 15\% and $5 \%$ lower than the existing methods $[15,22]$ such as $\mathrm{CE}$ with and without EEP-Kalman tracking, perfect channel estimation, FAH-LQE, and DMH-LQE

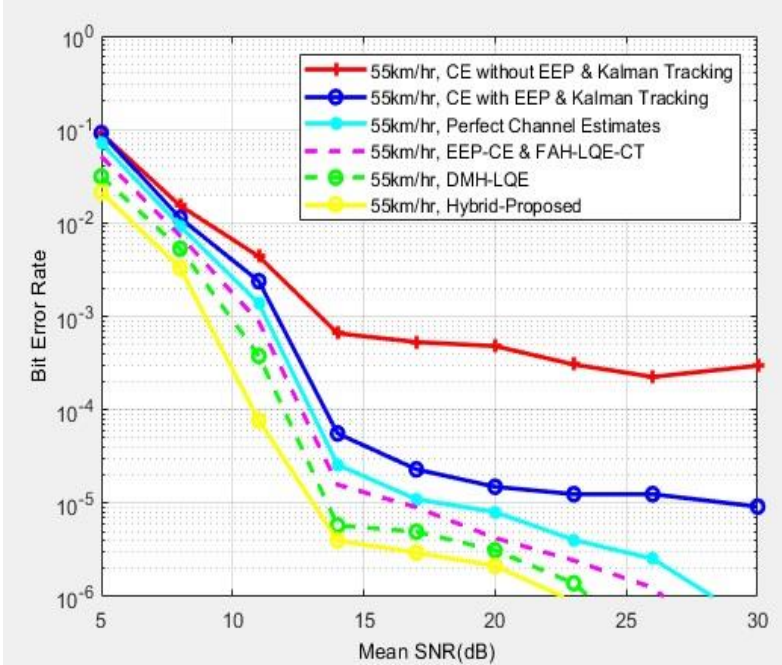

Figure. 5 BER performance of $(3 \times 2)$ MIMO-OFDM system with $\mu=55 \mathrm{~km} / \mathrm{hr}$ channels and $\mathrm{R}=600 \mathrm{kbps}$

respectively. The green colour represents the BER graph of the channel estimation by using Kalman tracking without considering the EEP method. Similarly, the sky blue colour indicates that Kalman tracking based channel estimation with EEP. The violet colour graph represents the channel estimation without using the Kalman filtering technique. The dark blue and yellow colour graphs represent the BER graphs of EEP-CE-FAH and DMH-LQE methods, respectively. The BER plot of the proposed Hybrid LQE method is represented in red colour. According to Fig. 4, the error rate is drastically reduced by using the proposed method.

In Fig. 5, the velocity of the user equipment (UE) such as mobile device can be considered as $55 \mathrm{~km} / \mathrm{hr}$. In this scenario the results are compared at SNR 15 and BER values of proposed algorithm (Hybrid LQE) is $22 \%, 10 \%, 7 \%, 6 \%$, and $3 \%$ lower than existing methods $[15,22]$ which is considered in the previous scenario.

The red colour represents the BER graph of the channel estimation by using kalman tracking without considering the EEP method. Similarly the dark blue colour indicates that, kalman tracking based channel estimation with EEP. The sky blue colour graph represents the channel estimation without using kalman filtering technique. The pink and green colour graphs represents the BER graphs of EEP-CE-FAH and DMH-LQE methods respectively. The BER plot of proposed Hybrid LQE method is represented in yellow colour. According to Fig. 5, the error rate is drastically reduced by using proposed method.

Fig. 6 shows the comparison results of Symbol Error rate (SER) performance. The SER is compared with phase noise, no compensation, and phase noise with compensation. The 5\% of SER has been reduced. 


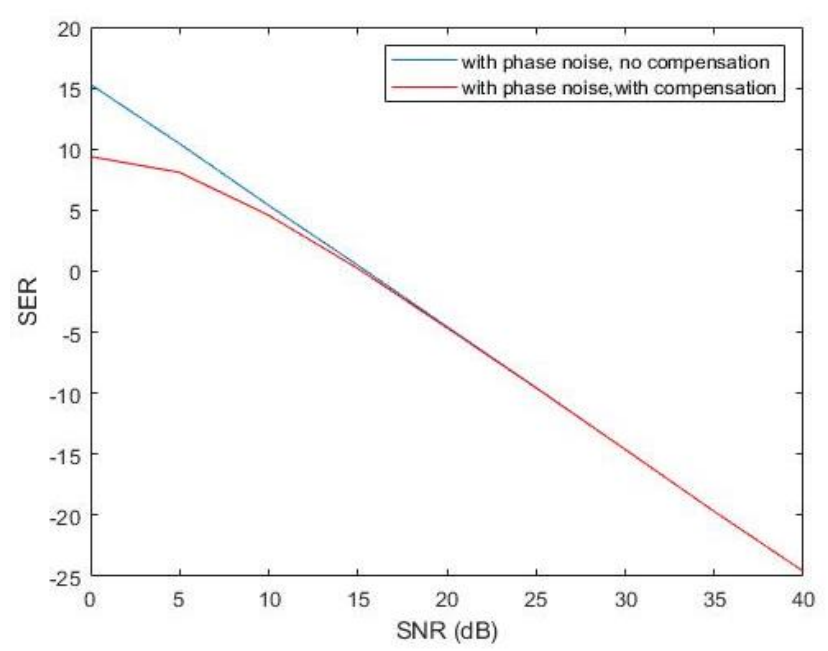

Figure. 6 Comparison SER performance results

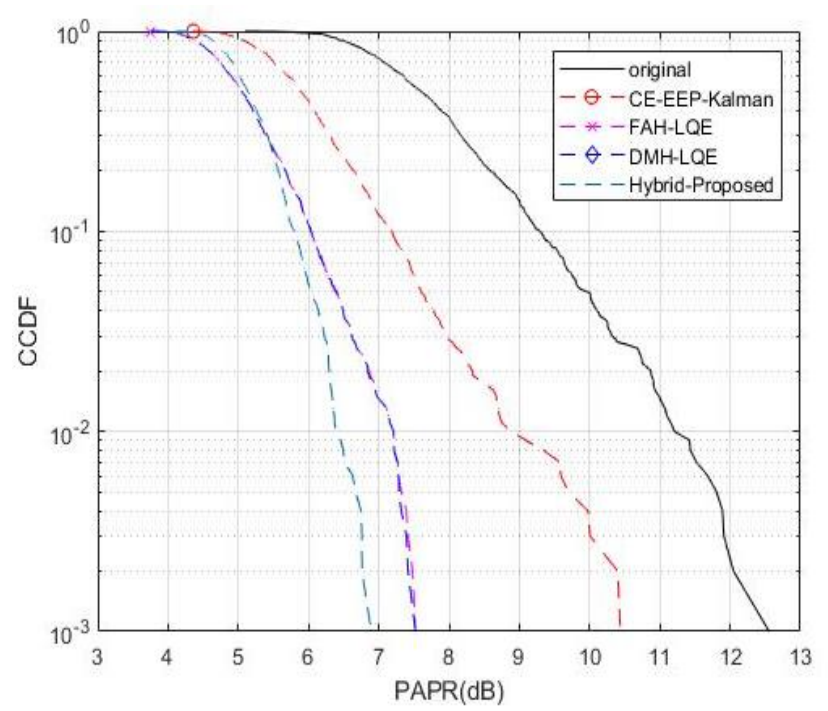

Figure. 7 Comparison results of PAPR vs CCDF

The blue line indicates the hybrid model without applying phase compensation and red line indicates the hybrid model with the phase compensation. By applying the phase compensation the SER has been reduced. Along with this, the transmission quality of the OFDM system is measured. The transmission quality of MIMO-OFDM system can be measured by using Peak to Average Power Ratio (PAPR) technique. The OFDM symbol is composed of multiple independently modulated sub- carrier signals superimposed. When the phases of the subcarriers are the same or close, the superimposed signal will be modulated by the same initial phase signal, resulting in greater instantaneous power. This can leads to increase the PAPR in MIMO-OFDM system. The lower PAPR can provide can provide larger coverage, better transmission quality, higher data rate and spectrum efficiency.
The PAPR of MIMO-OFDM signal can expressed as in Eq. (23).

$$
P A P R=\frac{\underset{0 \leq n \leq N-1}{\max }\left|x_{n}\right|^{2}}{E\left\{\left|x_{n}\right|^{2}\right\}}
$$

Where, $E\{\cdot\}$ represents the average value of the signal power.

The probability of PAPR is known as the complementary cumulative distribution function (CCDF), and expressed as in Eq. (24).

$$
\begin{aligned}
C C D F & =P_{r}\left\{P A P R>P A P R_{0}\right\} \\
& =1-\left(1-e^{-P A P R_{0}}\right)^{N}
\end{aligned}
$$

The PAPR comparison result is depicted in Fig. 7. The PAPR of proposed method is compared with other techniques such as CE-EEP with Kalman filter, FAH and DMH respectively. By using the proposed method, the PAPR has been reduced. The comparison results are made between without applying PAPR technique and with applying PAPR technique to the algorithms: CE-EEP kalaman filter, FAH-LQE, DMH-LQE and proposed Hybrid-LQE. By using proposed Hybrid-LQE algorithm with PAPR technique, 55\% reduced compared with without applying PAPR (original). Similarly, the proposed algorithm is compared with CE-EEP kalaman filter, the PAPR reduced by $35 \%$. The PAPR is reduced by $5 \%$ for both FAH and DMH-LQE algorithms [15, 22]. In Fig. 7, the block colour represents the original signal without applying PAPR technique. The red colour graph indicates that Kalman filter-based channel estimation method. Similarly pink and violet colour represents the FAH and DMH-LQE method respectively. Finally blue colour represents the PAPR result of proposed hybrid method.

\section{Conclusion}

This paper presented a hybrid LQE technique for Channel Estimation in MIMO-OFDM system. The noise covariance matrix $(\mathrm{Q})$ depends on the previous state values. In real-time scenarios these values are not always known. When the previous state values are known FAH-LQE method is used and the previous state values are unknown DMH-LQE is used. It is observed from simulation results, the proposed hybrid method reduces the BER, SER and PAPR values. At both scenarios $(55 \mathrm{~km} / \mathrm{h}$ and $115 \mathrm{~km} / \mathrm{h})$ with different SNRs ( $15 \mathrm{~dB}$ and $30 \mathrm{~dB})$, the proposed algorithms provides better results in terms of BER, SER and PAPR. In future, attempts made to 
apply deep learning based algorithms for the estimation of channel.

\section{Conflicts of Interest}

The authors declares that there is no conflict of interest'

\section{Author Contributions}

Chetana Reddy carried out the Linear-Quadratic Estimation (LQE) techniques for Channel Estimation in MIMO-OFDM system. A Hybrid LinearQuadratic Estimation (LQE) technique is developed by combining the Adaptive Hybrid - Linear Quadratic Estimation (FAH-LQE) and Discrete Multivariate Hybrid - Linear Quadratic Estimation (DMH-LQE) methods. Several tests carried out by considering the different velocities. The DFT based phase compensation model is incorporated.

Conceptualization, Chetana Reddy and Virendra Shete; methodology, Chetana Reddy; software and validation, Chetana Reddy; writing-review and editing, Chetana Reddy; supervision, Virendra Shete;

\section{Nomenclature}

\begin{tabular}{|c|c|}
\hline$G_{k} D_{k}$ and $X_{k}$ & State variables \\
\hline$A, B$ and $C$ & $\begin{array}{l}\text { Adoption/Channel transition } \\
\text { matrices }\end{array}$ \\
\hline$P_{k}$ & $\begin{array}{l}\text { Posterior estimate error } \\
\text { covariance matrix }\end{array}$ \\
\hline$P_{K}^{-}$ & $\begin{array}{l}\text { Prior estimate error covariance } \\
\text { matrix }\end{array}$ \\
\hline$Q$ & Process noise co variance matrix \\
\hline$\tilde{K}_{k}$ & Kalman gain \\
\hline$\sigma$ & $\begin{array}{l}\text { Noise co variance for process } \\
\text { and measurement models }\end{array}$ \\
\hline$I_{N_{t} N}$ & Identity matrix \\
\hline$X_{k p}$ & Predicted state matrix \\
\hline$P_{k p}$ & $\begin{array}{l}\text { Predicted process co-variance } \\
\text { matrix }\end{array}$ \\
\hline$\mu$ & Control variable matrix \\
\hline$w$ & Predicted state noise matrix \\
\hline$Z_{k}$ & Measurement noise \\
\hline$Y$ & Measurement of state \\
\hline$R$ & Sensor noise covariance matrix \\
\hline$D$ & Loss factor \\
\hline$T$ & Threshold \\
\hline$Q_{v}(n)$ & Previous state \\
\hline$Q_{u}(n)$ & Current state \\
\hline$s(n)$ & Transmitted signal \\
\hline$*$ & Multiplication \\
\hline$\odot$ & Convolution \\
\hline$\varnothing(n)$ & Phase noise \\
\hline$m(n)$ & Additive noise \\
\hline
\end{tabular}

$\begin{array}{cl}\text { EEP } & \text { Efficient Enlargement of pilot } \\ \text { LQE } & \text { Linear Quadric Estimation } \\ \text { CIR } & \text { Channel Impulse Response } \\ \text { UE } & \text { User Equipment } \\ \text { CE } & \text { Channel Estimation } \\ \text { CT } & \text { Channel Tracking } \\ \text { MSE } & \text { Mean Square Error } \\ \text { BER } & \text { Bit Error Rate } \\ \text { SER } & \text { Symbol Error Rate } \\ \text { PAPR } & \text { Peak to Average Power Ratio } \\ \text { CCDF } & \text { Complementary Cumulative } \\ & \text { Distribution Function } \\ \text { FAH } & \text { Fast Adaptive Hybrid } \\ \text { DMH } & \text { Discrete Multivariate Hybrid } \\ \text { ICI } & \text { Inter Carrier Interference } \\ \text { CSI } & \text { Channel State Information } \\ \text { CPE } & \text { Common Phase Error }\end{array}$

\section{References}

[1] A. S. Namitha and S. M. Sameer, "A bandwidth efficient selective mapping technique for the PAPR reduction in spatial multiplexing MIMOOFDM wireless communication system", Physical Communication, Vol. 25, No. 1, pp. 128-138, 2017.

[2] K. Dischant, S. Singh, R. Singh, and G. Sumeet, "OFDM modulation technique \& its applications: a review", In: Proc. of CCS, International Conference on Innovations in Computing, Landran Mohali, India, pp. 101-105, 2018.

[3] S. Nandi, A. Nandi, and N. N. Patha, "Performance analysis of Alamouti STBC MIMO OFDM for different transceiver system", In: Proc. of International Conference on Intelligent Sustainable Systems, Palladam, India, pp. 883-887, 2017.

[4] P. S. Kumar, M. Raju, and M. D. A. Iqbul, "Serial Concatenated Convolution Codes for Coded OFDM in Digital Audio Broadcasting Environment", In: Proc. of 2019 International Conference on Intelligent Sustainable Systems, Palladam, India, pp. 553-558, 2019.

[5] B. Ali and A. Hamze, "New fast time synchronization method for MIMO-OFDM systems", In: Proc. of 2018 11th IFIP Wireless and Mobile Networking Conference, Prague, Czech Republic, pp. 1-6, 2018.

[6] D. Xu, P. Ren, J. A. Ritcey, and Y. Wang, "Code-frequency block group coding for antispoofing pilot authentication in multi-antenna OFDM systems", IEEE Transactions on 
Information Forensics and Security, Vol. 13, No. 7, pp. 1778-1793, 2018.

[7] S. Stelios, F. Foukalas, and T. Khattab, "On the achievable rates of OFDM with common phase error compensation in phase noise channels", IEEE Transactions on Communications, Vol. 65, No. 8, pp. 3509-3521, 2017.

[8] S. Suraj, M. S. Kumar, M. Amrita, C. Sanjana, K. A. Jagannatham, and L. Hanzo, "Sparse doubly-selective channel estimation techniques for OSTBC MIMO-OFDM systems: A hierarchical Bayesian Kalman filter-based approach", IEEE Transactions on Communications, Vol. 68, No. 8 pp. 4844-4858, 2020.

[9] F. Ali, N. W. Hlaing, M. K. Haldar, and J. Rahebi, "Channel estimation for sparse channel OFDM systems using least square and minimum mean square error techniques", In: Proc. of International Conference on Engineering and Technology, Antalya, Turkey, pp. 1-5, 2017.

[10] K. Amit and I. Khurana. "Channel Estimation based on Kalman Filtering with BER Reduction in MIMO-OFDM Systems", International Journal of Computer Applications, Vol. 97, No. $1,2014$.

[11] C. D. kumar and B. Himanshu, "A novel channel estimation technique of MIMO-OFDM system based on Extended Kalman filter", In: Proc. of International Conference on Electronics and Communication Systems, Coimbatore, India, pp. 158-163, 2017.

[12] J. Lim, "Joint estimation of CFO and channel in OFDM systems with blind noise statistics", IETE Technical Review, Vol. 34, No. 5, pp. 491503, 2017.

[13] I. M. Ngebani, J. M. Chuma, I. Zibani, E. Matlotse, and K. Tsamaase, "Joint channel and phase noise estimation in MIMO-OFDM systems", In IOP Conference Series: Materials Science and Engineering, Vol. 198, No. 1, p. 012007, 2017.

[14] J. Bai, M. He, and C. Huang, "Self-interference channel estimation for full-duplex MIMOOFDM systems with phase noise", In: Proc. of IEEE International Conference on Communications Workshops, Kansas City, MO, USA, pp. 1-6, 2018.

[15] G. A. Devi and M. G. Babu, "A Robust Estimation and Tracking of Channel for Time and Frequency Selective MIMO-OFDM Systems", International Journal of Intelligent Engineering and Systems, Vol. 11, No. 3, 2018.

[16] H. Kaur, M. Khosla, and R. K. Sarin, "Channel estimation in MIMO-OFDM system: a review",
In: Proc. of International Conference on Electronics, Communication and Aerospace Technology, Coimbatore, India, pp. 974-980, 2018.

[17] O. Okoyeigbo, K. Okokpujie, E. N. Osaghae, C. U. Ndujiuba, O. Shobayo, and A. Jeremiah, "Comparative Study of MIMO-OFDM Channel Estimation in Wireless Systems", International Review on Modelling and Simulations, Vol. 11, No. 3, pp. 158-165, 2018.

[18] S. Pyla, K. P. Raju, and N. B. Subrahmanyam, "Performance analysis of adaptive filter channel estimated MIMO OFDM communication system", International Journal of Electrical \& Computer Engineering, Vol. 8, No. 5, pp. 20888708, 2018.

[19] P. De, "Reduced-Rank Filtering-Based Semiblind MIMO-OFDM Sparse Channel Estimation”, IEEE Systems Journal, Vol. 15, No. 1, pp. 1036-1047, 2021.

[20] S. Suraj, M. S. Kumar, A. Mishra, S. Chopra, K. A. Jagannatham, and L. Hanzo, "Sparse doublyselective channel estimation techniques for OSTBC MIMO-OFDM systems: A hierarchical Bayesian Kalman filter-based approach", IEEE Transactions on Communications, Vol. 68, No. 8, pp. 4844-4858, 2020.

[21] Z. Lu, H. Xu, D. Chen, and D. Qu, "Phase rotation to avoid imaginary interference leakage in multi-user MIMO-OFDM/OQAM systems", Wireless Personal Communications, Vol. 110, No. 4, pp. 1963-1984, 2020.

[22] G. A. Devi and M. G. Babu, "A Novel Estimation and Tracking of Channel for MIMOOFDM Systems", Journal of Advance Research in Dynamical \& Control Systems, Vol. 11, 2019. 